\title{
Abbreviations
}

$\begin{array}{ll}\text { AMLO } & \text { Andres Manuel López Obrador } \\ \text { CANACINTRA } & \begin{array}{l}\text { National Chamber of Manufacturing Industry (Cámara } \\ \text { Nacional de la Industria de Transformación) } \\ \text { Entrepreneurial Coordinating Council (Consejo } \\ \text { Coordinador Empresarial) }\end{array} \\ \text { CEREAL } & \begin{array}{l}\text { Reflection and Labor Action Center (Centro de Reflexión y } \\ \text { Acción Laboral) }\end{array} \\ \text { CMO } & \text { Mexican Classification of Occupations (Clasificación } \\ \text { COPARMEX } & \begin{array}{l}\text { Mexicana de Ocupaciones) } \\ \text { Patronal de la República Mexicana) }\end{array} \\ \text { CROC } & \begin{array}{l}\text { Revolutionary Confederation of Workers and Peasants } \\ \text { (Confederación Revolucionaria de Obreros y Campesinos) }\end{array} \\ \text { CTC } & \text { Confederation of Workers and Peasants (Confederación de } \\ \text { CTM } & \text { Trabajadores y Campesinos) } \\ \text { Confederation of Mexican Workers (Confederación de } \\ \text { FAT } & \text { Trabajadores de México) } \\ \text { FCAB } & \text { Authentic Labor Front (Frente Auténtico del Trabajo) } \\ \text { federal conciliation and arbitration board (junta federal } \\ \text { de conciliación y arbitraje) } \\ \text { FDI }\end{array}$


ILOGB International Labor Office Governing Body

IMF

International Monetary Fund

IMSS

Mexican Social Security System (Instituto Mexicano del Seguro Social)

INEGI National Institute of Statistics, Geography, and Information (Instituto Nacional de Estadística, Geografía e Informática)

INFONAVIT National Workers' Housing Fund (Instituto del Fondo Nacional de la Vivienda para los Trabajadores)

IRB institutional review board

ISCO-88 International Standard Classification of Occupations-[19]88

ISO

ISPT

$\mathrm{J}-1$

International Organization for Standardization

payroll tax (Impuesto Sobre Productos del Trabajo)

jackets one (production line)

$\mathrm{J}-2$

JIT

LCAB

jackets two (production line)

just-in-time

local conciliation and arbitration board (junta local de conciliación y arbitraje)

MFA Multi-Fibre Arrangement

MNR1 Major Regional Newspaper One (center-left perspective)

MORENA Movement for National Renovation (Movimiento de la Regeneración Nacional)

$M R N_{2}$

NAFTA

Major Regional Newspaper Two (center-right perspective)

NGO

OAS

North American Free Trade Agreement

nongovernmental organization

PAN

Organization of American States

PNR

National Action Party (Partido Acción Nacional)

National Revolutionary Party (Partido Revolucionario

Nacional)

PRD Democratic Revolution Party (Partido de la Revolución

Democrática)

PRI Institutional Revolutionary Party (Partido Revolucionario Institucional)

SAMs standard allotted minutes

SAR Savings for Retirement System (Sistemas de Ahorro para

el Retiro)

SPC statistical process control

UNT National Workers' Union (Unión Nacional de

Trabajadores)

WTO World Trade Organization 


\section{We Are in This Dance Together of}


